\title{
Impact of Direct Acting Antiviral (DAA) Treatment on Glucose Metabolism and Reduction of Pre-diabetes in Patients with Chronic Hepatitis C
}

\author{
Philip Weidner ${ }^{1}$, Dominik Boettche ${ }^{1}$, Thomas Zimmerer ${ }^{2}$, Elke Burgermeister ${ }^{1}$, Andreas Teufel ${ }^{1}$, Matthias P.A. Ebert ${ }^{1}$, \\ Christoph Antoni ${ }^{1}$
}

1) Dept. of Medicine II,

Medical Faculty Mannheim, Heidelberg University, 68167 Mannheim, Germany

2) Clinic of Internal Medicine, Center of Gastroenterology, East-Tallinn Central Hospital, 10138 Tallinn, Estland

\begin{abstract}
Background \& Aim: With the development of direct acting antiviral agents (DAA) chronic hepatitis C virus (HCV) infection has become curable in most patients. Since HCV infection is known to have direct and/or indirect effects on glucose metabolism, successful HCV treatment may have an impact in reducing glucose level, pre-diabetes, the need of treatment for diabetes, and ultimately diabetes-associated morbidity. We investigated the association of DAA treatment and glucose metabolism in the context of development or resolution of hepatic fibrosis in a large cohort of HCV- infected patients.

Methods: In this retrospective single-center observational study, we investigated 281 patients receiving alloral DAA therapy for fasting plasma glucose, $\mathrm{HbA1c}$, liver enzymes and general clinical chemistry, measured during a 52-week follow-up. In addition, elastography, FIB-4- and APRI-calculation were used to assess hepatic fibrosis non-invasively.

Results: Successful elimination of HCV through DAA treatment was associated with a significant drop in fasting glucose level and a reduced rate of impaired fasting plasma glucose (FPG). Interestingly, this metabolic change was BMI-independent. In addition, long-term glucose levels also decreased after successful DAA treatment. A significant APRI-score reduction was associated with a persistent improvement of FPG. However, DAA did not have an impact on glucose metabolism in patients suffering from liver cirrhosis.

Conclusion: This study highlights the beneficial impact of successful HCV therapy on glucose metabolism and identifies patients with liver cirrhosis as a collective in need of intensified surveillance with regard to diabetes progression despite $\mathrm{HCV}$ eradication.
\end{abstract}

Key words: Hepatitis C - direct acting antiviral - diabetes - metabolism.

Abbreviations: DAA: direct acting antiviral agents; FPG: fasting plasma glucose; HCV: hepatitis C virus; IFG: impaired fasting glucose; SVR: sustained virological response.

\section{INTRODUCTION}

Received: 13.04.2018

Accepted: 12.08 .2018
Chronic infection with hepatitis $\mathrm{C}$ virus ( $\mathrm{HCV}$ ) is one of the major causes of chronic liver disease, cirrhosis and its complications as well as hepatocellular carcinoma. Affecting approximately 170 million people worldwide with an increasing morbidity and mortality, $\mathrm{HCV}$ is a major issue for global health $[1,2]$. Yielding sustained virological response (SVR) rates between $79 \%$ and $100 \%$ for the combination of Simeprevir and Sofosbuvir [3],
$93 \%$ to $99 \%$ for Sofosbuvir plus Ledipasvir $[4,5], 91 \%$ to $96 \%$ in compensated cirrhosis for the regimen containing Ombitasvir, Paritaprevir, Ritonavir and Dasabuvir [6], and $92 \%$ to $100 \%$ for Daclatasvir and Sofosbuvir [2], the phase II and III studies demonstrated chronic HCV infection to be a curable disease in most patients.

Achieving SVR leads to a decrease in mortality and morbidity in line with a reduction of the complications associated with liver disease progression, such as cirrhosis, liver failure, or hepatocellular carcinoma [7]. Comparing paired liver biopsies obtained before and after achieved SVR by interferon-based therapies, an improvement in hepatic inflammation was demonstrated in about $90 \%$ of patients [7]. This finding was accompanied by a reduction of histologic fibrosis scores. However, the reported rate of fibrosis regression ranges from $25 \%$ [8] to 56\% [9] with lower rates found in larger study populations. Using non-invasive assessments of 
hepatic fibrosis, this change seems to be slow, leading to a small reduction of the number of patients progressing to cirrhosis [10]. Adding the DAAs Boceprevir and Telaprevir to the eradication regimen, results in a rapid and significant drop in serum markers of liver fibrosis [11]. Beside promoting hepatic fibrosis and carcinogenesis, chronic inflammation caused by the HCV has been associated with an impairment of the liver central role in glucose homeostasis, leading to an increased incidence of prediabetes, diabetes and hepatic steatosis [12, 13]. Eradication of the HCV virus, achieved by interferonbased therapies, was first linked to an improvement of the markers of glucose metabolism in a hospital-based study in a relatively small Chinese population [14]. In line with this observation, Pavone et al. [15] were the first to describe a rapid improvement of fasting plasma glucose (FPG) following SVR achieved by interferon-free regimens. Hitherto, these data have been further substantiated by additional smaller reports on this issue [16-19]. However, correlation to body mass index (BMI) and/or liver cirrhosis remained unclear in most of these studies.

Therefore, we analyzed these aspects of DAA-based hepatitis $\mathrm{C}$ therapy on the metabolic function of the liver in a retrospective single-center observational study.

\section{PATIENTS AND METHODS}

\section{HCV cohort}

This retrospective single-center observational study group comprised 281 patients treated with DAA \pm Ribavirin between
March 2013 and June 2016 in the outpatient department of the Mannheim University Medical Center. The analysis included treatment-naïve and treatment-experienced patients with and without cirrhosis. HCV genotypes included genotypes $1-5$. Follow-up data were available 4 to 52 weeks post-treatment. There were no specific inclusion or exclusion criteria for this analysis. Table I describes patient baseline characteristics by genotype. There were no significant differences regarding gender $(\mathrm{p}=0.8827), \mathrm{BMI}(\mathrm{p}=0.8897)$ or frequency of liver cirrhosis $(\mathrm{p}=0.8391)$ for the different genotypes. The baseline prevalence of diabetes mellitus type 2 (DMT2) was $10 \%$ $(n=28)$. Further data regarding DMT2 patients is provided in Suppl. Table I. Impaired fasting plasma glucose (IFG) could be detected in 22 of the 129 patients (17\%) with sufficient data on FPG.

\section{Treatment regimens}

Therapeutic regimens included Daclatasvir (Bristol-Myers Squibb S.r.l., Contrada Fontana del Ceraso, Italy), Ledipasvir (Gilead Sciences Ireland UC, Carrigtohill, Ireland), Simeprevir (Janssen-Cilag, Borgo San Michele, Italy), Sofosbuvir (Gilead Sciences Ireland UC, Carrigtohill, Ireland), the combination of Paritaprevir, Ritonavir and Ombitasvir (AbbVie Deutschland, Ludwigshafen, Germany) with or without Dasabuvir (AbbVie Deutschland, Ludwigshafen, Germany) and Velpatasvir (Gilead Sciences Ireland UC, Carrigtohill, Ireland) and Ribavirin (Roche Pharma, Grenzach-Wyhlen, Germany). All medications were administered as recommended by the manufacturer. The

Table I. Baseline Characteristics (available information*)

\begin{tabular}{|c|c|c|c|c|c|c|c|c|c|c|}
\hline \multicolumn{6}{|l|}{ Median age [years] (281) } & \multicolumn{5}{|c|}{56} \\
\hline \multicolumn{6}{|c|}{ Mean BMI $\left[\mathrm{kg} / \mathrm{m}^{2}\right](205)\left(\mathrm{SD}^{1}\right)$} & \multicolumn{5}{|c|}{$26(5.11)$} \\
\hline \multirow{2}{*}{\multicolumn{3}{|c|}{ Baseline $\log 10 \mathrm{HCV}$ RNA titre $[\mathrm{IU} / \mathrm{ml}]$ (122) }} & \multicolumn{3}{|c|}{ Mean $\left(\mathrm{SD}^{1}\right)$} & \multicolumn{5}{|c|}{$2(3)$} \\
\hline & & & \multicolumn{3}{|c|}{ Median $\left(\mathrm{IQR}^{2}\right)$} & \multicolumn{5}{|c|}{$1(2)$} \\
\hline \multirow[t]{2}{*}{ Genotype $\left(281^{\star}\right)$} & & $\mathrm{n}(\%)$ & $1^{3}(\%)$ & 1a $(\%)$ & $1 \mathrm{a} / \mathrm{b}(\%)$ & $1 \mathrm{~b}(\%)$ & $2(\%)$ & $3 a(\%)$ & 4 & $5 \mathrm{a}$ \\
\hline & & & $203(72)$ & $71(25)$ & $2(1)$ & $118(42)$ & $8(3)$ & $53(19)$ & $16(6)$ & $1(1)$ \\
\hline \multirow[t]{2}{*}{$\operatorname{Sex}\left(281^{\star}\right)$} & $\mathrm{m}$ & $154(55)$ & $102(36)$ & $36(13)$ & $2(1)$ & $59(21)$ & $3(1)$ & $39(14)$ & $9(3)$ & - \\
\hline & $\mathrm{f}$ & $127(45)$ & $101(36)$ & $35(13)$ & - & $59(21)$ & $5(2)$ & $14(5)$ & $7(2)$ & $1(1)$ \\
\hline \multicolumn{2}{|l|}{ Prediabetes $\left(129^{*}\right)$} & $22(17)$ & $15(12)$ & $2(2)$ & - & $11(9)$ & $1(1)$ & $3(2)$ & $3(2)$ & - \\
\hline \multicolumn{2}{|l|}{ Diabetes $\left(281^{\star}\right)$} & $28(10)$ & $21(7)$ & $9(3)$ & - & $9(3)$ & $1(1)$ & $3(1)$ & $3(1)$ & - \\
\hline \multicolumn{2}{|l|}{ Cirrhosis $\left(267^{\star}\right)$} & $98(37)$ & $75(28)$ & $28(10)$ & - & $44(16)$ & $4(1)$ & $16(6)$ & $4(1)$ & - \\
\hline \multirow[t]{3}{*}{ Child-Pugh Class $\left(95^{\star}\right)$} & A & $83(87)$ & $63(66)$ & $23(24)$ & - & $38(40)$ & $4(4)$ & $14(15)$ & $3(3)$ & - \\
\hline & B & $8(8)$ & $7(7)$ & $3(3)$ & - & $2(2)$ & - & - & $1(1)$ & - \\
\hline & $\mathrm{C}$ & $4(4)$ & $3(3)$ & - & - & $3(3)$ & - & $1(1)$ & - & - \\
\hline \multicolumn{2}{|l|}{ Mean MELD $\left(98^{*}\right)$} & 10 & 10 & 10 & - & 10 & 16 & 9 & 9 & - \\
\hline \multirow[t]{4}{*}{ Fibrosis $\left(210^{*}\right)$} & F0-1 & $71(34)$ & $55(26)$ & $20(10)$ & $2(1)$ & $28(13)$ & $2(1)$ & $10(5)$ & $2(1)$ & $1(1)$ \\
\hline & $\mathrm{F} 2$ & $40(19)$ & $26(12)$ & $4(2)$ & - & $21(10)$ & $1(1)$ & $7(3)$ & $7(3)$ & - \\
\hline & F3 & $35(17)$ & $25(12)$ & $10(5)$ & - & $15(7)$ & - & $8(4)$ & $2(1)$ & - \\
\hline & F4 & $64(30)$ & $51(24)$ & $22(10)$ & - & $26(12)$ & $3(1)$ & $9(4)$ & $1(1)$ & - \\
\hline \multirow[t]{4}{*}{ Treatment history $\left(123^{*}\right)$} & Naïve & $77(63)$ & $35(28)$ & $12(10)$ & - & $21(17)$ & $4(3)$ & $7(6)$ & $1(1)$ & - \\
\hline & Int $^{4}$ & $13(11)$ & $10(8)$ & $1(1)$ & $2(2)$ & $7(6)$ & - & $2(2)$ & - & - \\
\hline & Int $+\mathrm{Rv}^{5}$ & $56(46)$ & $40(33)$ & $9(7)$ & - & $26(21)$ & $1(1)$ & $5(4)$ & $3(2)$ & - \\
\hline & $\mathrm{Rv}+\mathrm{PI}^{6}$ & $19(15)$ & $16(13)$ & $3(2)$ & - & $9(7)$ & - & - & - & - \\
\hline
\end{tabular}

${ }^{*}$ Values marked with a star represent the number of patients with available information regarding the parameter in question. Percentages are calculated using this value. ${ }^{1}$ Standard deviation; ${ }^{2}$ Inter quartile range; ${ }^{3}$ Containing patients without further subgenotyping; ${ }^{4}$ Interferon; ${ }^{5}$ Ribavirin;

${ }^{6}$ Protease inhibitor 
therapeutic regimen was selected by a board of hepatologists based on the current state of scientific information or followed the recommendations of the European Association for the Study of the Liver and the German Gastroenterological Association DGVS, once available. The most frequent therapeutic regimens used consisted of Sofosbuvir and Ledipasvir with or without Ribavirin (26\%), Sofosbuvir and Daclatasvir with or without Ribavirin (26\%), but a significant subset was treated with Ombitasvir, Paritaprevir, Ritonavir and Dasabuvir (17\%).

\section{Quantification of liver fibrosis}

Hepatic fibrosis was estimated using the FIB-4 test combining alanine- and aspartate aminotransferase (ASAT) levels, platelet count and age according to Sterling et al. [20], the ASAT to platelet ratio index (APRI) according to Wai et al. [21] or transient elastography measured by FibroScan ${ }^{\circledast}$ or Acoustic Radiation Force Impulse (ARFI) imaging. Cutoffs used were $\leq 7 / 1.215$ (F0-1), 7.1-9.5/1.216-1.54 (F2), 9.6-12.5/1.55-1.94 (F3), $\geq 12.6 / 1.94$ (F4) for FibroScan and ARFI, respectively. Sert et al. [22] described a 70\% higher APRI value in insulinresistant patients compared with healthy controls. Therefore, we examined whether a reduction of the APRI score by this amount could be associated with an improvement of glucose metabolism in the same individual.

\section{Evaluation of metabolic changes}

Fasting plasma glucose levels were determined within one hour using lithium heparin as an anticoagulant. Impaired fasting glucose was defined as a blood glucose level ranging from $100-125 \mathrm{mg} / \mathrm{dl}$.

\section{Statistical analysis}

Statistical analysis was conducted using SAS 9.4 and Graphpad Prism 4.0. The Shapiro-Wilk test was performed to assess normal distribution, a two-sided Student's $t$-Test was used for normally distributed data, Sign- and the Wilcoxon signed-rank test for non-parametric data. To compare different therapeutic regimens and genotypes, One-Way ANOVA and Holm-Sidak's multiple comparisons test were employed. A p-value less than 0.05 was regarded as statistically significant in all analyses.

\section{Ethical approval}

All procedures performed in studies involving human participants were in accordance with the ethical standards of the institutional and/or national research committees and with the 1964 Helsinki declaration and its later amendments or comparable ethical standards, as confirmed by the Ethics Committee of the Medical Faculty Mannheim, Germany. Written informed consent was obtained from all individual participants included in the study.

\section{RESULTS}

\section{DAA treatment efficacy, virological outcome}

In our "real world" cohort, general SVR rates were high, with an overall SVR4 rate of $99 \%(n=281)$ and an overall SVR12 rate of $94 \%(n=265)$. Regarding the treatment failure group, male gender, overweight, IFG and patients with genotype 3a were overrepresented (Suppl. Table II).

\section{Effect of HCV treatment on plasma glucose levels}

The effect of the SVR on the liver's metabolic function was assessed by determining the FPG level before treatment, at the end of the treatment as well as during an up to 48 weeks follow-up period. Sufficient baseline and follow-up data was available for 129 patients. SVR was associated with a significant reduction in fasting glucose level $(p<0.0001, n=129)$. This effect was maintained until SVR24 $(\mathrm{p}=0.0005, \mathrm{n}=109)$. At SVR48, however, the mean FPG value regained the starting level (Fig. 1a, Table II).

The effect on FPG became apparent in patients with impaired fasting glucose levels (data not shown) or manifest DMT2 (Table II) as well as in patients with normal fasting glucose levels (data not shown). In all cases in which a reduction in fasting plasma glucose was shown, a significant drop of serum transaminases became apparent (ASAT: $\mathrm{p}<0.000181 \pm 6 \mathrm{U} / \mathrm{l}$ vs. $33 \pm 3 \mathrm{U} / \mathrm{l}$, ALAT: $<<0.0001106 \pm 9$ $\mathrm{U} / \mathrm{l}$ vs. $37 \pm 3 \mathrm{U} / \mathrm{l}, \mathrm{n}=98$ ).

Impaired fasting glucose was present in $17 \%(n=22)$ of the patients before the initiation of therapy. After completion of therapy, IFG was observed only in $6 \%(n=8)$ of the patients $(\mathrm{p}=0.0017, \mathrm{n}=129)$. During the same period of observation, body mass index (BMI) did not change (Fig. 1b).
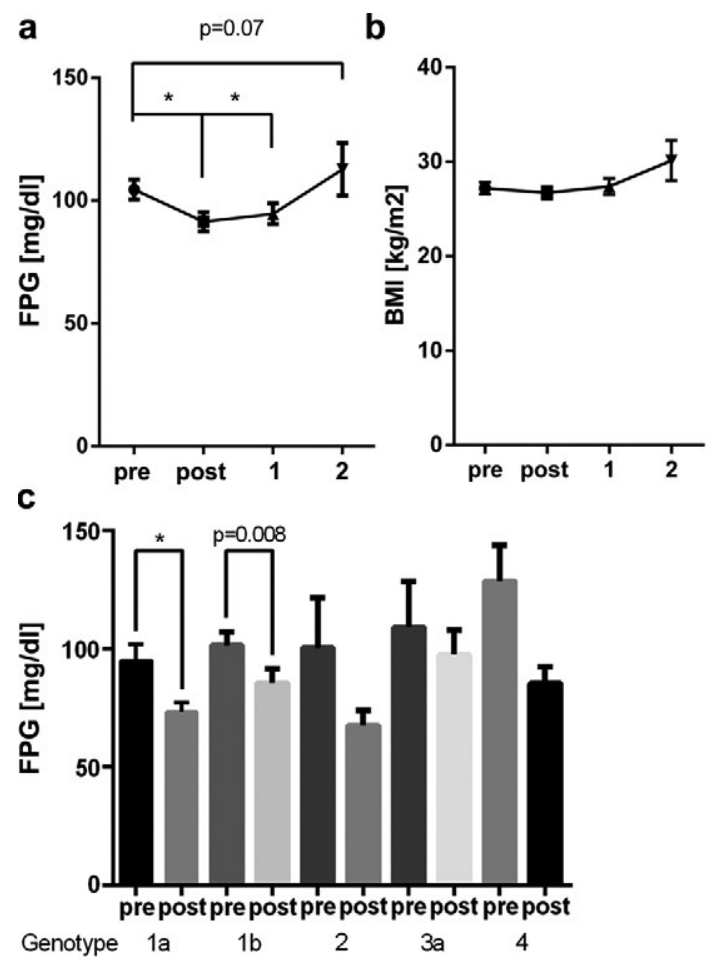

Fig. 1. Impact of SVR on fasting plasma glucose levels. SVR leads to a consistent drop in FPG levels, sustained until SVR24 (a). Absolute values in $\mathrm{mg} / \mathrm{dl} \pm$ SEM post-treatment (SVR4), at SVR 24 (1) and 48 (2). SVR is not associated with a significant change in BMI (b). Absolute values in $\mathrm{kg} / \mathrm{m} 2 \pm \mathrm{SEM}$. A significant reduction in plasma fasting glucose levels could be shown in patients infected with genotype $1 \mathrm{a}$ and $1 \mathrm{~b}(\mathrm{c})$. 
Table II. Results of the investigations in our patients

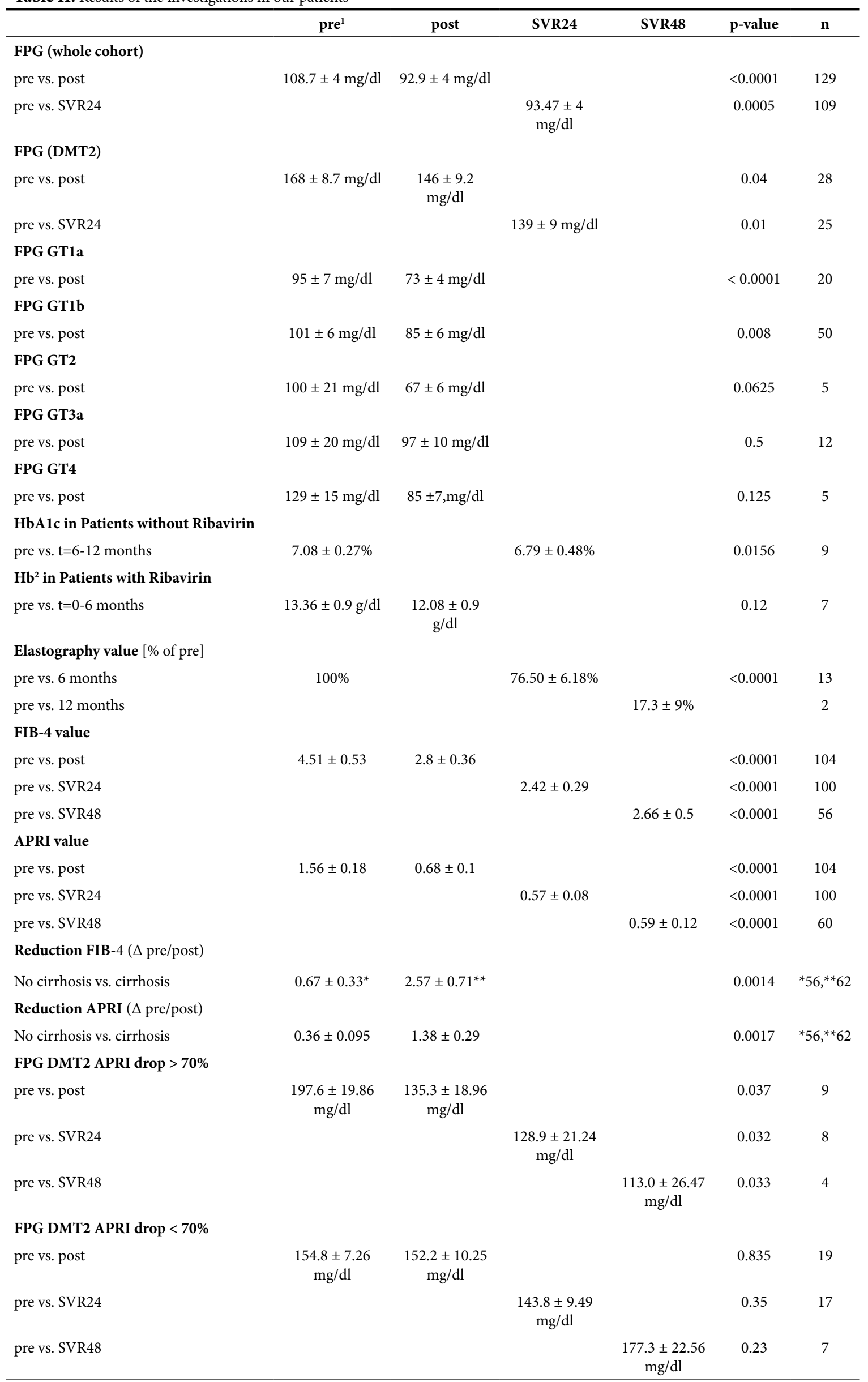




\begin{tabular}{|c|c|c|c|c|c|c|}
\hline \multicolumn{7}{|c|}{ Table II (continued) } \\
\hline & pre $^{1}$ & post & SVR24 & SVR48 & p-value & $\mathbf{n}$ \\
\hline \multicolumn{7}{|c|}{ FPG IFG APRI drop $>70 \%$} \\
\hline pre vs. post & $\begin{array}{c}125.2 \pm 7 \\
\mathrm{mg} / \mathrm{dl}^{*}\end{array}$ & $\begin{array}{c}93.60 \pm 9 \\
\mathrm{mg} / \mathrm{dl}{ }^{* *}\end{array}$ & & & 0.0007 & ${ }^{\star} 13,{ }^{* *} 12$ \\
\hline pre vs. SVR24 & & & $\begin{array}{c}84.00 \pm 5 \\
\mathrm{mg} / \mathrm{dl}\end{array}$ & & 0.0003 & 6 \\
\hline \multicolumn{7}{|c|}{ FPG IFG APRI drop $<70 \%$} \\
\hline pre vs. post & $\begin{array}{c}138.9 \pm 16.6 \\
\mathrm{mg} / \mathrm{dl}\end{array}$ & $\begin{array}{c}104.1 \pm 14.4 \\
\mathrm{mg} / \mathrm{dl}\end{array}$ & & & 0.006 & 9 \\
\hline pre vs. SVR24 & & & $\begin{array}{c}107 \pm 10.4 \\
\mathrm{mg} / \mathrm{dl}\end{array}$ & & 0.059 & 8 \\
\hline pre vs. SVR48 & & & & $\begin{array}{c}120.3 \pm 21.8 \\
\mathrm{mg} / \mathrm{dl}\end{array}$ & & 4 \\
\hline \multicolumn{7}{|c|}{ FPG no IFG/DMT2 APRI drop > 70\% } \\
\hline pre vs. post & $\begin{array}{c}82.77 \pm 1.4 \\
\mathrm{mg} / \mathrm{dl}\end{array}$ & $\begin{array}{c}72.06 \pm 2 \\
\mathrm{mg} / \mathrm{dl}\end{array}$ & & & $<0.0001$ & 35 \\
\hline \multicolumn{7}{|c|}{ FPG no IFG/ DMT2 APRI drop $<70 \%$} \\
\hline pre vs. post & $79.56 \pm 2 \mathrm{mg} / \mathrm{dl}$ & $\begin{array}{c}71.82 \pm 3 \\
\mathrm{mg} / \mathrm{dl}\end{array}$ & & & 0.0055 & 39 \\
\hline
\end{tabular}

An obvious trend towards a reduction of fasting glucose level could be found in all HCV genotypes (Table II). In genotype $1 \mathrm{a}$ and $1 \mathrm{~b}$, this difference was significant (Table II). Again, BMI remained unchanged ( $p>0.5$, Suppl. Fig. 1a-c).

\section{Effect of $\mathrm{HCV}$ treatment on long-term parameters of glucose metabolism (HbA1c)}

With regard to changes in long-term parameters of glucose metabolism, $\mathrm{HbAlc}$ was assessed only in patients with manifest DMT2. Of the 28 patients with DMT2, baseline values were available from 22 patients, 12 of whom received a follow-up 6 months after therapy termination. The 12- and 18-month data were available for 15 individuals and 24-month follow-up data for 9 patients.

As for the plasma glucose levels, HbA1c demonstrated a significant decrease following $\mathrm{HCV}$ eradication in patients with manifest DMT2 ( $p=0.0367 n=12$, Fig. 2a). In this subgroup, the decrease of HbAlc was accompanied by a non-significant reduction in BMI immediately after therapy (pre vs. post: $30.35 \pm 2 \mathrm{~kg} / \mathrm{m}^{2} \mathrm{n}=17$ vs. $\left.27.18 \pm 2 \mathrm{~kg} / \mathrm{m}^{2} \mathrm{p}=0.1, \mathrm{n}=5\right)$. In the non-cirrhotic subgroup, we observed a significant decrease of the HbAlc values up to 12 months after therapy (pre vs. $t=0$ 6 months: $\mathrm{p}=0.09 \mathrm{n}=6$; pre vs. $\mathrm{t}=6-12$ months: $\mathrm{p}=0.03 \mathrm{n}=6$; Fig. 2b). However, in patients with liver cirrhosis this effect was not observed (pre vs. $\mathrm{t}=0-6$ months: $\mathrm{p}=0.4 \mathrm{n}=7$; pre vs. $t=6-12$ months: $p=0.1 n=9$; Fig. $2 c$ ). The highest impact on $\mathrm{HbA1c}$ improvement had the presence of steatosis. In those cases, a trend for prolonged reduction of $\mathrm{HbAlc}$ became apparent ( $n=8$, Fig. 2d), whereas there was no effect in patients with DMT2 without steatosis $(\mathrm{n}=11$, Fig. 2e). As Ribavirinassociated hemolytic anemia may lead to an artificially low measurement of HbA1c [23], we performed the same analysis in patients not receiving Ribavirin. In this subpopulation, we also detected a significant reduction in HbA1c 6-12 months after therapy (Suppl. Figure 1d, Table II). In patients, receiving

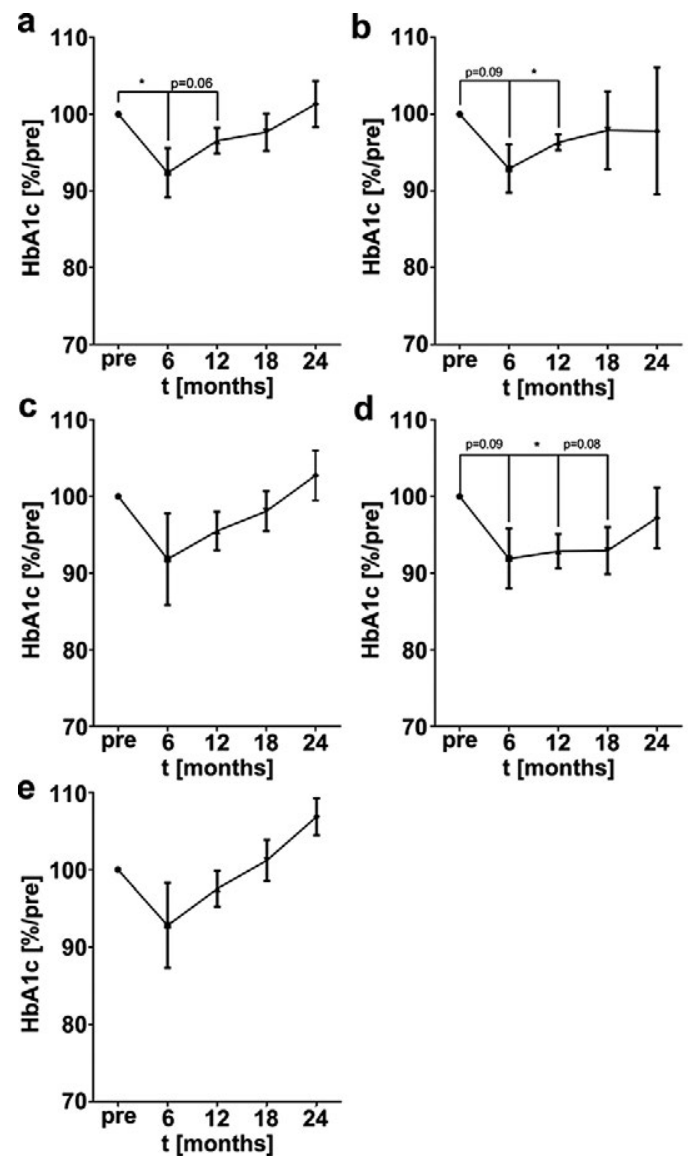

Fig. 2. Effect of HCV treatment on $\mathrm{HbAlc}$. The relative $\mathrm{HbAl}$ c values \pm SEM, normalized on the pretreatment value (pre) at 6, 7-12 (12), 1318 (18) and 19-24 (24) months post treatment of all patients suffering from DMT2 (a), DMT2 without liver cirrhosis (b) and patients suffering from DMT2 and manifest cirrhosis (c). In the presence of steatosis $(d)$, a trend for a prolonged effect could be shown $(6: n=6,12$ : $\mathrm{n}=5,18: \mathrm{n}=5,24: \mathrm{n}=4)$, while there was no effect detectable in patients without steatosis (e) $(6: n=6,12: n=8,18: n=8,24: n=3)$. 
Ribavirin no significant change in HbAlc could be detected (Suppl. Figure 1e), albeit a non-significant decrease in $\mathrm{Hb}$ was observed (Suppl. Figure 1f, Table II). Notably, in this subgroup 5 of 7 patients suffered from liver cirrhosis.

\section{Effect of DAA treatment on hepatic fibrosis}

To investigate whether a correlation of glucose metabolism and liver fibrosis can be detected, we investigated the course of development or reversal of fibrosis during and after DAA treatment. Therefore, we applied the non-invasive fibrosis assessment modalities FIB-4 and APRI and performed elastography before treatment initiation in 55\% (154/281) of all patients.

Performing elastography 4 to 12 weeks after termination of therapy, no difference to the baseline elasticity values was detected $(p=0.203$, mean difference of the Fibroscan value pre and post treatment: $2.29 \pm 4.23 ; \mathrm{n}=31$ ). However, after 6 and 12 months, a significant reduction was observed (pre vs. 6 months: $\mathrm{p}<0.0001$, $n=13$; pre vs. 12 months: $n=2$; Fig. 3a). Overall, $40 \%$ of the patients were shown to have a decrease in liver fibrosis, another $48 \%$ were stable with respect to fibrosis and only $12 \%$ progressed within 12 months after treatment (Fig. 3b). Of note, in patients with genotype 3a no significant reduction in any fibrosis score could be detected.

Evaluating FIB-4 value pre- and post-treatment, a decrease in the frequency of calculated advanced fibrosis by $19 \%$ was observed, accompanied by a significant reduction in absolute FIB-4 values (pre vs. post: $\mathrm{p}<0.0001, \mathrm{n}=104$; pre vs. SVR24: $\mathrm{p}<0.0001, \mathrm{n}=100$; pre vs. SVR $48: \mathrm{p}<0.0001, \mathrm{n}=56$ ) (Fig. $3 \mathrm{c}$, Table II). The same effect was demonstrated calculating the APRI score pre- and post-treatment (Fig. 3d, Table II).

Regarding the reduction of the two fibrosis scores, the effect was significantly more pronounced in cirrhotic livers (Fig. 3e\&f, Table II).

\section{Correlation of plasma fasting glucose and liver fibrosis in patients with DMT2}

Fasting plasma glucose levels of patients with manifest DMT2 and IFG were significantly changed depending on the underlying fibrosis status (Fig. 4). In patients with manifest diabetes and a decrease in APRI of more than $70 \%$, a significant decrease of FPG was observed throughout treatment and follow-up (Fig. 4a), while this decrease did not become apparent in patients with an APRI drop smaller than $70 \%$ (Fig. 4b, Table II).

\section{Correlation of plasma fasting glucose and liver fibrosis in patients with IFG}

Similar changes were observed in patients with impaired fasting glucose and an APRI drop of more than $70 \%$, where a decrease in FPG was observed up to week 24 post treatment (pre vs. post: $\mathrm{p}=0.0007, \mathrm{n}=13$ vs. $\mathrm{n}=12$; pre vs. SVR24: $p=0.0003, n=13$ vs. $n=6$; Fig. 4 c). However, in patients with IFG and an APRI drop of less than 70\% no significant effect on the FPG level was observed at SVR24 and SVR48 (Fig. 4d, Table II).

Correlation of plasma fasting glucose and liver fibrosis in patients with preserved glucose metabolism

In patients not suffering from diabetes or IFG, the changes described above were only moderate and occurred immediately after therapy termination (Fig. 4e, f, Table II). In these patients, FPG even slightly increased back to base levels after termination of treatment. a

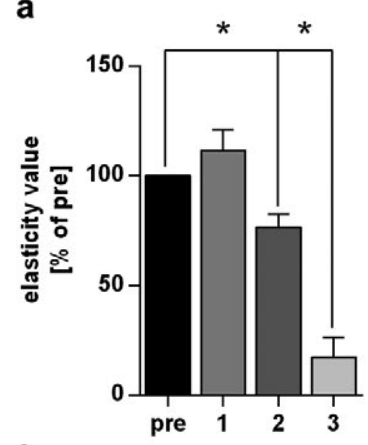

C
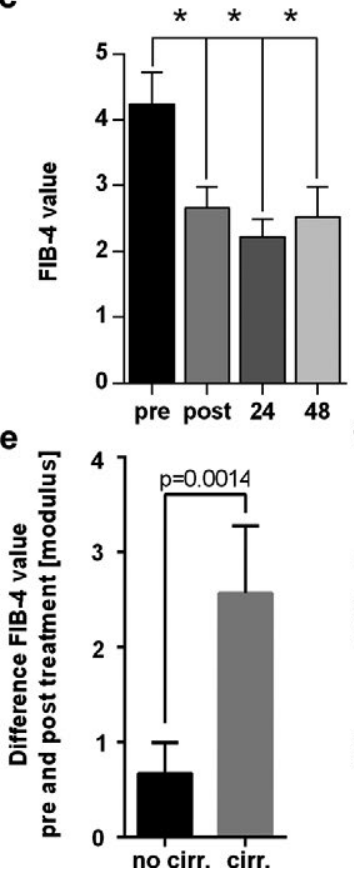

b

d
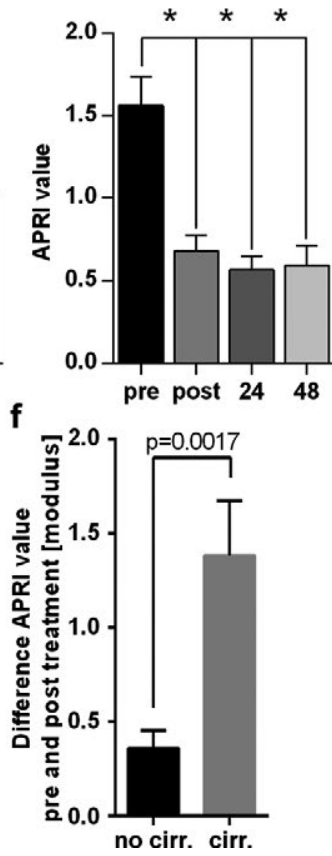

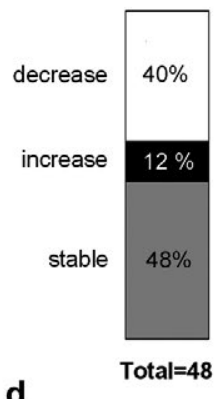

Fig. 3. SVR leads to a significant reduction of hepatic stiffness, preceded by a significant drop of FIB-4 and APRI values. (a) The elasticity values measured by ARFI and Fibroscan in percent of the value attained before initiation of treatment. First measurement (1) was performed within 6 weeks after termination of therapy, second measurement (2) within 6 months and third measurement (3) within 12-14 months. (b) A course of hepatic fibrosis following SVR. (c-d) SVR led to a significant and lasting reduction in FIB-4 and APRI values: depicted are the absolute values calculated pre- and posttreatment at SVR 24 and $48 \pm$ SEM. (e-f). The reduction of the FIB- 4 and APRI values was more pronounced in patients with liver cirrhosis.

\section{DISCUSSION}

Hepatocytes are crucial for maintenance of plasma glucose homeostasis. In a constant adjustment between glucose production and utilization via the gluconeogenic and glycolytic pathways, these cells are at the center of plasma glucose level regulation. Throughout the past decade, multiple molecular mechanisms of the HCV virus interfering at different levels with glucose metabolism have been established. Among these 


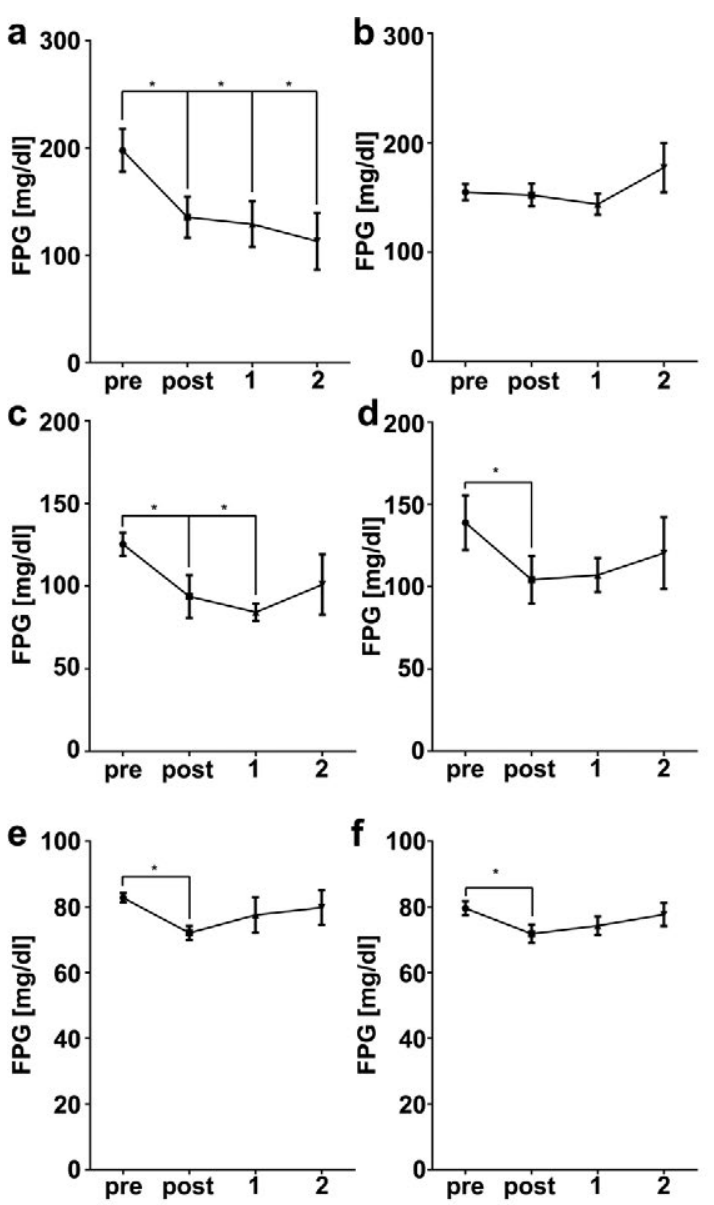

Fig. 4. Correlation of plasma fasting glucose and liver fibrosis. The absolute FPG values \pm SEM in patients with a drop in APRI $>70 \%$ $(\mathrm{a}, \mathrm{c}, \mathrm{e}),<70 \%(\mathrm{~b}, \mathrm{~d}, \mathrm{f})$ and DMT2 (a, b), IFG (c,d) and with normal fasting plasma glucose levels $(e, f)$, pre and post treatment as well as at SVR24 (1) and 48 (2).

are effects of HCV replication on cellular glucose uptake through down-regulation of cell surface expression of glucose transporter 2 (GLUT2) [24] or hepatic gluconeogenesis via an NS5A-mediated, FoxO1-dependent pathway [25]. These molecular changes translate into a close link between HCV and DMT2. The large cross-sectional NHANES III study, surveying 9841 U.S. adults, demonstrated that HCV-positive U.S. patients above 40 years had a threefold increased risk for diabetes mellitus [26]. Numerous other studies have further substantiated these findings [27, 28]. An IFG rate of $18 \%$ detected in our patients is in line with these reports.

The availability of DAA-based therapies started a new era of HCV treatment with high efficacy and acceptable side effects even under "real-world" conditions [29]. In line with these published data, we achieved in our cohort overall SVR4 and SVR12 rates of $98 \%$ and $94 \%$, respectively. Likewise, with $98 \%$ SVR12 rate being accomplished by using a regimen based on Daclatasvir or Ledipasvir (SVR12: 93\% $\mathrm{n}=70$ ), the treatment of our patients with DAA was very successful. Treatment failure most often occurred in the genotype 3a subgroup, which has already been shown to be the subpopulation most difficult to treat with a DAA-containing regimen [30-32].

Given the well-established correlation between HCV and glucose metabolism and the high rate of viral clearance utilizing the novel DAAs, we aimed to investigate the impact of DAA treatment and viral clearance on glucose metabolism. Our findings regarding reduced plasma fasting glucose levels and $\mathrm{HbAlc}$ values were in line with published literature on this issue [16-19]. In addition to these reports, we demonstrated in our more comprehensive cohort that these differences in glucose metabolism were independent of changes in body mass index (BMI). Our cohort comprised a constant high number of overweight patients and a mean BMI of $25.27 \mathrm{~kg} /$ $\mathrm{m}^{2}$. Thus, the observed changes in glucose levels might be an effect of HCV clearance $[24,25]$. The observation that even in patients with preserved glucose metabolism a reduction of FPG can be obtained, indicates a subclinical insulin resistance associated with $\mathrm{HCV}$ infection. This conclusion is in line with reports of reduced plasma insulin and HOM-IR following SVR [17]. Given our study's prolonged observation period, we were able to characterize this effect as only transient. While in all subgroups a significantly reduced FPG level could be seen only up to SVR24, patients with DMT2 and a $>70 \%$ drop of the APRI value achieved a sustained improvement of FPG. As both scores highly weight transaminases in their calculation, these results may stress the importance of hepatic inflammation in HCV-induced insulin resistance, rather than the presence of fibrosis, especially in light of the observation of the delayed improvement in hepatic fibrosis, determined by elastography. Of note, 4 (14\%) patients with DMT2 developed a new insulin dependency in the immediate aftermath of the therapy, of whom no patient achieved a $>70 \%$ drop of APRI at the end of the therapy.

Finally, we were concerned that Ribavirin may induce hemolytic anemia, subsequently lowering $\mathrm{HbAlc}$ artificially. This was ruled out by analyzing HbAlc values in Ribavirinfree patients only, yielding similar results. In addition, FPG levels, not affected by hemolytic anemia, reflected HbAlc measurements.

Our data on changes in glucose metabolism in patients with DMT2 and liver cirrhosis are of considerable interest regarding their clinical implications. In contrast to other publications, we demonstrate that this subgroup of patients does not benefit from DAA treatment with respect to improvement of glucose levels. One reason for this lack of benefit might be that an important part of the pro-diabetic potential of chronic HCVinfection is due to peripheral insulin resistance caused e.g. by direct impairment of glucose transporter 4 expression by the HCV core protein in skeletal muscle and adipose tissue [33]. In cirrhotic patients, muscle tissue may exhibit impaired glucose storage because of reduced insulin sensitivity independent of HCV infection [34]. In addition, a reduced hepatic insulin clearance contributes to hyperinsulinemia and concomitant insulin resistance as well as increased plasma levels of insulincounteracting hormones and a lack of liver-derived humoral factors with insulin-like activity, e.g. insulin-like growth factors I and II [35]. However, Gitto et al. [36] described a significant reduction in serum insulin levels after DAA-based SVR in a mainly cirrhotic group, while being unable to detect an effect on plasma glucose levels. Thus, one might explain the reduced responsiveness to DAA treatment of cirrhotic patients with regard to glucose metabolism by either a reduced effect on the peripheral glucose uptake or the decreased hepatic production 
of insulin-like factors. Our data contributes to the growing evidence that DAA-based HCV eradication might result in a decreased cardiovascular risk both by the resolution of hepatic and systemic HCV-toxicity as indicated e.g. by a reduction of carotid atherosclerosis [37].

Given the retrospective approach of the current analysis, several limitations of this study have to be taken into account. The non-invasive assessment of hepatic fibrosis is limited by the lack of more specific laboratory tests such as ELF-test or Pro-C3 assessment and the absence of elastography measurements in all patients. The same applies for parameters of insulin resistance. The heterogeneity study population limits its explanatory power with regard to the possibility of correction for potential confounders such as genotype and the treatment regimen used.

\section{CONCLUSION}

Direct acting antiviral treatment was confirmed to be highly effective in a large "real-world" cohort of HCV patients and exhibited beneficial effects beyond viral elimination especially on glucose metabolism. This effect was independent of patients' BMI. However, DAA did not have an impact on glucose metabolism in patients suffering from liver cirrhosis.

\section{Conflicts of interest: None declared.}

Authors' contributions: P.W., D.B.,T.Z.,C.A.: data-collection; P.W.,T.Z., CA: data analysis and interpretation; P.W., E.B., A.T.: drafting of the manuscript; E.B., A.T., M.P.A.E., C.A: critical revision of the manuscript for important intellectual content.

Supplementary material: To access the supplementary material visit the online version of the J Gastrointestin Liver Dis at http://www. jgld.ro/wp/archive/y2018/n3/a13 and http://dx.doi.org/10.15403/ jgld.2014.1121.273.daa

\section{REFERENCES}

1. Global alert and response (GAR). Hepatitis C. 2002. Accessed: 01.08.2016. Available at: http://www.who.int/csr/disease/hepatitis/ whocdscsrlyo2003/en/index4.html

2. Sulkowski MS, Gardiner DF, Rodriguez-Torres M, et al. Daclatasvir plus sofosbuvir for previously treated or untreated chronic HCV infection. N Engl J Med 2014;370:211-221. doi:10.1056/NEJMoa1306218

3. Lawitz E, Sulkowski MS, Ghalib R, et al. Simeprevir plus sofosbuvir, with or without ribavirin, to treat chronic infection with hepatitis $\mathrm{C}$ virus genotype 1 in non-responders to pegylated interferon and ribavirin and treatment-naive patients: the COSMOS randomised study. Lancet 2014;384:1756-1765. doi:10.1016/S0140-6736(14)61036-9

4. Afdhal N, Zeuzem S, Kwo P, et al. Ledipasvir and sofosbuvir for untreated HCV genotype 1 infection. N Engl J Med 2014;370:1889-1898. doi:10.1056/NEJMoa1402454

5. Kowdley KV, Gordon SC, Reddy KR, et al. Ledipasvir and sofosbuvir for 8 or 12 weeks for chronic HCV without cirrhosis. N Engl J Med 2014;370:1879-1888. doi:10.1056/NEJMoa1402355

6. Khullar V, Firpi RJ. Hepatitis C cirrhosis: New perspectives for diagnosis and treatment. World J Hepatol 2015;7:1843-1855. doi:10.4254/wjh. v7.i14.1843
7. Lee YA, Friedman SL. Reversal, maintenance or progression: what happens to the liver after a virologic cure of hepatitis C? Antiviral Res 2014;107:23-30. doi:10.1016/j.antiviral.2014.03.012

8. Poynard T, McHutchison J, Manns M, et al. Impact of pegylated interferon alfa-2b and ribavirin on liver fibrosis in patients with chronic hepatitis C. Gastroenterology 2002;122:1303-1313. doi:10.1053/ gast.2002.33023

9. Maylin S, Martinot-Peignoux M, Moucari R, et al. Eradication of hepatitis $\mathrm{C}$ virus in patients successfully treated for chronic hepatitis C. Gastroenterology 2008;135:821-829. doi:10.1053/j. gastro.2008.05.044

10. Poynard T, Moussalli J, Munteanu M, et al. Slow regression of liver fibrosis presumed by repeated biomarkers after virological cure in patients with chronic hepatitis C. J Hepatol 2013;59:675-683. doi:10.1016/j.jhep.2013.05.015

11. Haseltine EL, Penney MS, George S, Kieffer TL. Successful treatment with telaprevir-based regimens for chronic hepatitis $\mathrm{C}$ results in significant improvements to serum markers of liver fibrosis. J Viral Hepat 2015;22:701-707. doi:10.1111/jvh.12382

12. Mohamed HR, Abdel-Azziz MY, Zalata KR, Abdel-Razik AM. Relation of insulin resistance and liver fibrosis progression in patients with chronic hepatitis C virus infection. Int J Health Sci (Qassim) 2009;3:177186.

13. Pais R, Rusu E, Zilisteanu D, et al. Prevalence of steatosis and insulin resistance in patients with chronic hepatitis $\mathrm{B}$ compared with chronic hepatitis $\mathrm{C}$ and non-alcoholic fatty liver disease. Eur J Intern Med 2015;26:30-36. doi:10.1016/j.ejim.2014.12.001

14. Qing S, Ji D, Li B, et al. Improvement of glucose and lipid metabolism with pegylated interferon-a plus ribavirin therapy in Chinese patients chronically infected with genotype $1 \mathrm{~b}$ hepatitis C virus. Ann Saudi Med 2015;35:293-297. doi:10.5144/0256-4947.2015.293

15. Pavone $\mathrm{P}$, Tieghi T, d'Ettorre G, et al. Rapid decline of fasting glucose in HCV diabetic patients treated with direct-acting antiviral agents. Clin Microbiol Infect 2016;22:462 e1-e3. doi:10.1016/j.cmi.2015.12.030

16. Hum J, Jou JH, Green PK, et al. Improvement in Glycemic Control of Type 2 Diabetes After Successful Treatment of Hepatitis C Virus. Diabetes Care 2017;40:1173-1180. doi:10.2337/dc17-0485

17. Salomone F, Catania M, Montineri A, et al. Hepatitis $C$ virus eradication by direct antiviral agents improves glucose tolerance and reduces postload insulin resistance in nondiabetic patients with genotype 1. Liver Int 2018;38:1206-1211. doi:10.1111/liv.13669

18. Ikeda A, Ikeda K, Takai A, et al. Hepatitis C Treatment with Sofosbuvir and Ledipasvir Accompanied by Immediate Improvement in Hemoglobin A1c. Digestion 2017;96:228-230. doi:10.1159/000484237

19. Ciancio A, Bosio R, Bo S, et al. Significant improvement of glycemic control in diabetic patients with $\mathrm{HCV}$ infection responding to directacting antiviral agents. J Med Virol 2018;90:320-327. doi:10.1002/ jmv.24954

20. Sterling RK, Lissen E, Clumeck N, et al. Development of a simple noninvasive index to predict significant fibrosis in patients with HIV/ HCV coinfection. Hepatology 2006;43:1317-1325. doi:10.1002/hep.21178

21. Wai CT, Greenson JK, Fontana RJ, et al. A simple noninvasive index can predict both significant fibrosis and cirrhosis in patients with chronic hepatitis C. Hepatology 2003;38:518-526. doi:10.1053/jhep.2003.50346

22. Sert A, Pirgon O, Aypar E, Yilmaz H, Dundar B. Relationship between aspartate aminotransferase-to-platelet ratio index and carotid intimamedia thickness in obese adolescents with non-alcoholic fatty liver disease. J Clin Res Pediatr Endocrinol 2013;5:182-188. doi:10.4274/ Jcrpe.891 
23. Robertson M. Artificially low HbA1c associated with treatment with ribavirin. BMJ 2008;336:505. doi:10.1136/bmj.39457.828287.47

24. Kasai D, Adachi T, Deng L, et al. HCV replication suppresses cellular glucose uptake through down-regulation of cell surface expression of glucose transporters. J Hepatol 2009;50:883-894. doi:10.1016/j.jhep.2008.12.029

25. Deng L, Shoji I, Ogawa W, et al. Hepatitis C virus infection promotes hepatic gluconeogenesis through an NS5A-mediated, FoxO1-dependent pathway. J Virol 2011;85:8556-8568. doi:10.1128/JVI.00146-11

26. Mehta SH, Brancati FL, Sulkowski MS, Strathdee SA, Szklo M, Thomas DL. Prevalence of type 2 diabetes mellitus among persons with hepatitis C virus infection in the United States. Ann Intern Med 2001;33:1554. doi:10.1053/jhep.2001.0103306le01

27. Mason AL, Lau JY, Hoang N, et al. Association of diabetes mellitus and chronic hepatitis C virus infection. Hepatology 1999;29:328-333. doi:10.1002/hep.510290235

28. Hammerstad SS, Grock SF, Lee HJ, Hasham A, Sundaram N, Tomer Y. Diabetes and Hepatitis C: A Two-Way Association. Front Endocrinol (Lausanne) 2015;6:134. doi:10.3389/fendo.2015.00134

29. Hezode C, Fontaine H, Dorival C, et al. Effectiveness of telaprevir or boceprevir in treatment-experienced patients with HCV genotype 1 infection and cirrhosis. Gastroenterology 2014;147:132-142.e4. doi:10.1053/j.gastro.2014.03.051

30. Nelson DR, Cooper JN, Lalezari JP, et al. All-oral 12-week treatment with daclatasvir plus sofosbuvir in patients with hepatitis $C$ virus genotype 3 infection: ALLY-3 phase III study. Hepatology 2015;61:1127-1135. doi: $10.1002 /$ hep. 27726
31. Jacobson IM, Gordon SC, Kowdley KV, et al. Sofosbuvir for hepatitis C genotype 2 or 3 in patients without treatment options. N Engl J Med 2013;368:1867-1877. doi:10.1056/NEJMoa1214854

32. Lawitz E, Mangia A, Wyles D, et al. Sofosbuvir for previously untreated chronic hepatitis C infection. N Engl J Med 2013;368:1878-1887. doi:10.1056/NEJMoa1214853

33. Garcia-Compean D, Gonzalez-Gonzalez JA, Lavalle-Gonzalez FJ, Gonzalez-Moreno EI, Villarreal-Perez JZ, Maldonado-Garza HJ. Current Concepts in Diabetes Mellitus and Chronic Liver Disease: Clinical Outcomes, Hepatitis C Virus Association, and Therapy. Dig Dis Sci 2016;61:371-380. doi:10.1007/s10620-015-3907-2

34. Selberg O, Burchert W, vd Hoff J, et al. Insulin resistance in liver cirrhosis. Positron-emission tomography scan analysis of skeletal muscle glucose metabolism. J Clin Invest 1993;91:1897-1902. doi:10.1172/JCI116407

35. Nolte W, Hartmann H, Ramadori G. Glucose metabolism and liver cirrhosis. Exp Clin Endocrinol Diabetes 1995;103:63-74. doi:10.1055/s-0029-1211331

36. Gitto S, Cicero AFG, Loggi E, et al. Worsening of Serum Lipid Profile after Direct Acting Antiviral Treatment. Ann Hepatol 2018;17:64-75. doi:10.5604/01.3001.0010.7536

37. Petta S, Adinolfi LE, Fracanzani AL, et al. Hepatitis C virus eradication by direct-acting antiviral agents improves carotid atherosclerosis in patients with severe liver fibrosis. J Hepatol 2018;69:18-24. doi:10.1016/j. jhep.2018.02.015 
Supplementary Table I Baseline Characteristics of Patients with Type 2 Diabetes

\begin{tabular}{|c|c|c|c|c|c|c|c|c|c|c|c|c|c|c|c|c|c|c|c|}
\hline \multicolumn{8}{|c|}{ Characteristic (available information*) } & & & & & & & & & & & & \\
\hline \multicolumn{8}{|l|}{ Median age [years] (28*) } & \multicolumn{12}{|c|}{62,5} \\
\hline \multicolumn{8}{|c|}{ Mean BMI [kg/m2] (21*) (STD) } & \multicolumn{12}{|c|}{$30,35(7,4)$} \\
\hline \multirow{2}{*}{\multicolumn{2}{|c|}{ Genotype (28*) }} & \multicolumn{2}{|c|}{$n(\%)$} & \multicolumn{2}{|c|}{$1^{1}(\%)$} & \multicolumn{2}{|c|}{$1 a(\%)$} & \multicolumn{2}{|c|}{$1 a / b(\%)$} & \multicolumn{2}{|c|}{$16(\%)$} & \multicolumn{2}{|c|}{$2(\%)$} & \multicolumn{2}{|c|}{$3 a(\%)$} & \multicolumn{2}{|c|}{4} & \multicolumn{2}{|c|}{$5 \mathbf{a}$} \\
\hline & & & & 21( & (75) & 9( & (32) & & & $9(3$ & & & (4) & & 11) & & 11) & & - \\
\hline \multirow[t]{2}{*}{$\operatorname{sex}\left(28^{\star}\right)$} & $m$ & \multicolumn{2}{|c|}{$16(57)$} & \multicolumn{2}{|c|}{$10(35)$} & \multicolumn{2}{|c|}{$4(14)$} & \multicolumn{2}{|c|}{-} & \multicolumn{2}{|c|}{$5(18)$} & \multicolumn{2}{|c|}{-} & \multicolumn{2}{|c|}{$3(11)$} & \multicolumn{2}{|c|}{$3(11)$} & \multicolumn{2}{|c|}{-} \\
\hline & $\boldsymbol{f}$ & \multicolumn{2}{|c|}{$12(43)$} & \multicolumn{2}{|c|}{$11(39)$} & \multicolumn{2}{|c|}{$5(18)$} & \multicolumn{2}{|c|}{-} & \multicolumn{2}{|c|}{$4(14)$} & \multicolumn{2}{|c|}{$1(4)$} & \multicolumn{2}{|c|}{-} & & - & & - \\
\hline Cirrhosis (27*) & & 19 & (70) & 13( & (48) & 5( & (19) & & & $6(2$ & & & (4) & & 11) & & (7) & & - \\
\hline Child-Pugh Class (19*) & $A$ & 14 & (74) & 10( & (53) & 5( & 26) & & & $4(2$ & 21) & & (5) & & 11) & & (5) & & - \\
\hline & $B$ & 4( & 21) & $2(1$ & 11) & - & - & & & 1( & & & - & & (5) & & (5) & & - \\
\hline & C & 1 & (5) & 1( & (5) & - & - & & & 1( & & & - & & 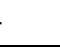 & & - & & - \\
\hline Medication (28*) & & $1^{2}$ & $\mathrm{O}^{3}$ & I & $\mathrm{O}$ & I & $\mathrm{O}$ & I & $\mathrm{O}$ & I & O & I & $\mathrm{O}$ & I & $\mathrm{O}$ & I & $\mathrm{O}$ & I & $\mathrm{O}$ \\
\hline & & $\begin{array}{c}8 \\
(29)\end{array}$ & $\begin{array}{c}20 \\
(71)\end{array}$ & $\begin{array}{c}5 \\
(18)\end{array}$ & $\begin{array}{r}16 \\
(57)\end{array}$ & $\begin{array}{c}1 \\
(4)\end{array}$ & $\begin{array}{c}8 \\
(29)\end{array}$ & - & - & $\begin{array}{c}4 \\
(14)\end{array}$ & $\begin{array}{c}5 \\
(18)\end{array}$ & - & $\begin{array}{c}1 \\
(4)\end{array}$ & $\begin{array}{c}2 \\
(7)\end{array}$ & $\begin{array}{c}1 \\
(4)\end{array}$ & $\begin{array}{c}1 \\
(4)\end{array}$ & $\begin{array}{c}2 \\
(7)\end{array}$ & - & - \\
\hline DAA Therapy + Ribavir & $\operatorname{in}\left(28^{\star}\right)$ & 11 & (40) & $5(1$ & 18) & 3( & 11) & & & 2( & & & - & & 11) & & 11) & & - \\
\hline
\end{tabular}

\footnotetext{
*Values marked with a star represent the number of patients with available information regarding the parameter in question. Percentages are calculated using this value.

${ }^{1}$ Containing patients without further subgenotyping

${ }^{2}$ Insulin

${ }^{3}$ Oral diabetic drug
} 

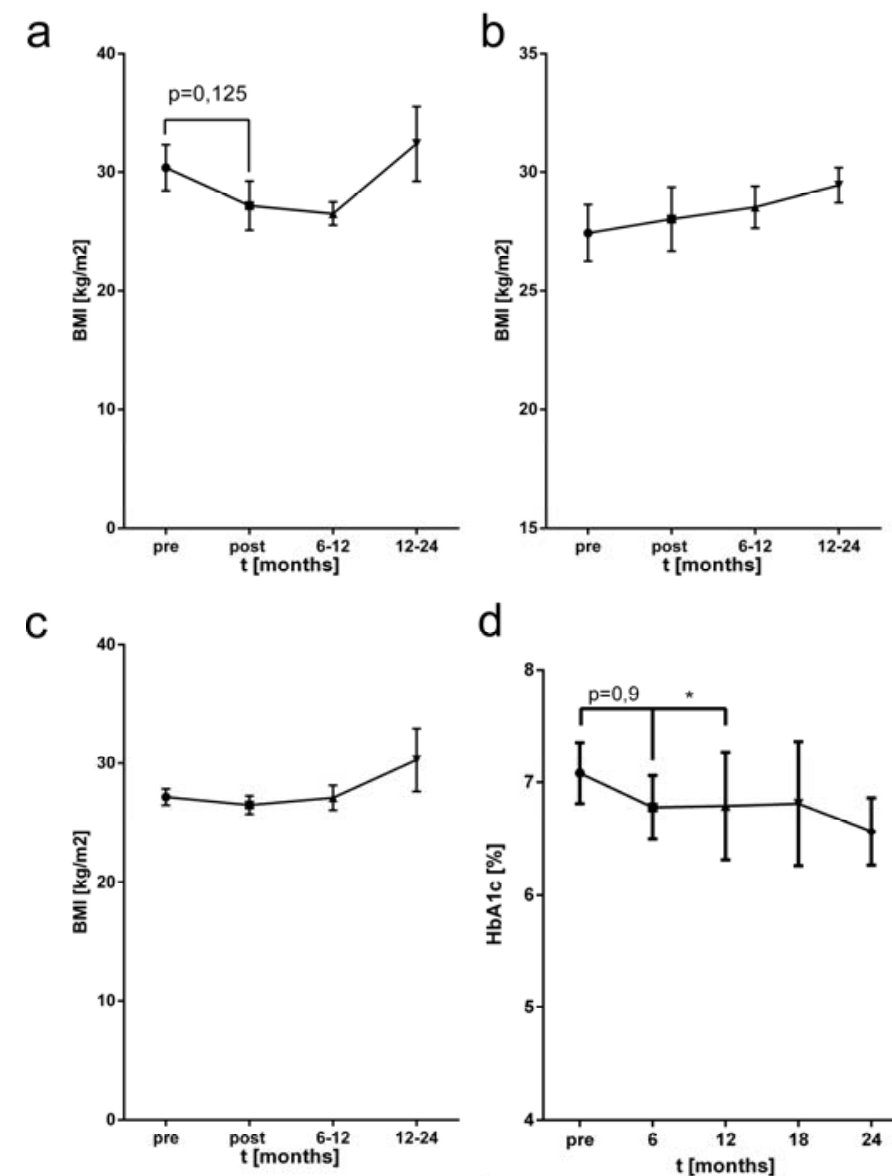

d
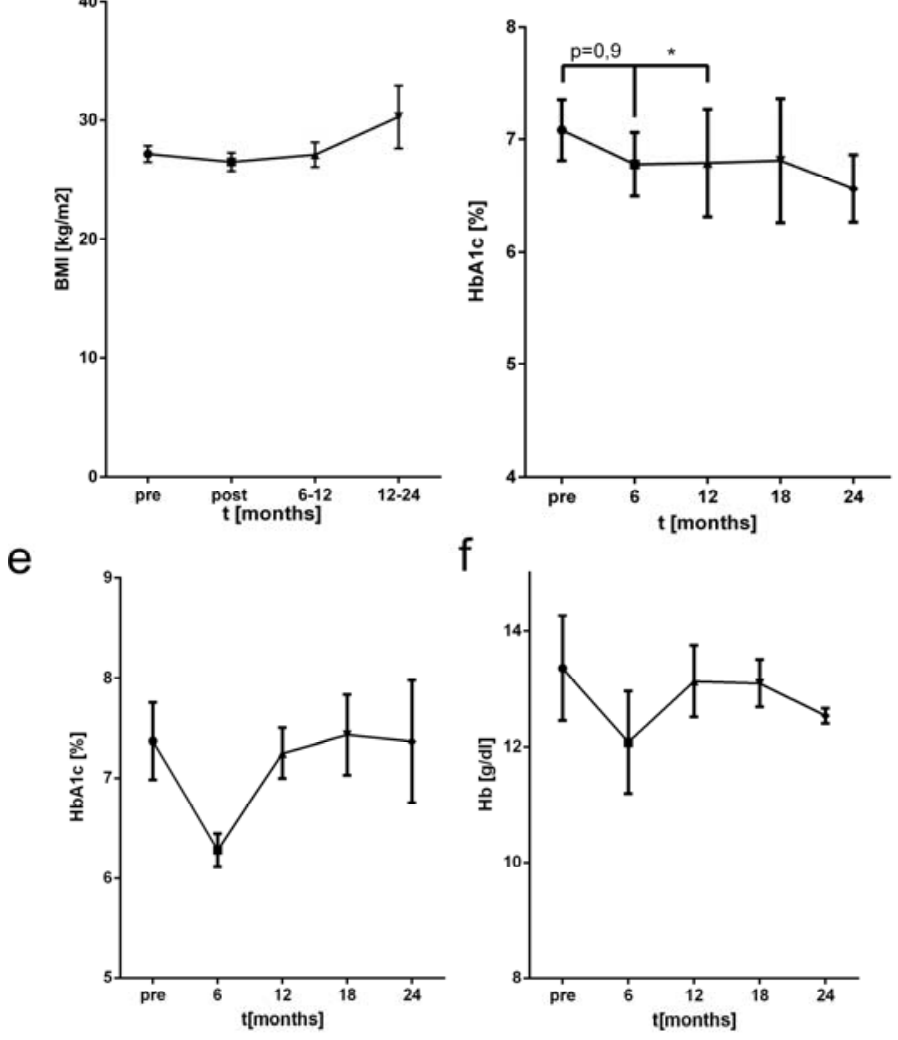

Supplementary Figure 1: Correlation of BMI and characteristics of glucose metabolism: Depicted are the absolute BMI values in $\mathrm{kg} / \mathrm{m}^{2}$ in patients with DMT2 (a), IFG (b) and with normal FPG levels (c), pre and post treatment as well as after 6 to 12 and 12 to 24 months (x-axis). Data was available for $11,5,3$, and 10 patients with diabetes (pre, post, 6-12, 12-24), 9, 6, 6 and 3 patients with IFG and 72, 37, 26 and 13 patients with preserved glucose tolerance. A trend for a change in BMI was observed in patients with DMT2, (a). SVR is associated with a significant reduction of $\mathrm{HbA1c}$ in patients not receiving Ribavirin (d) and receiving a Ribavirin-containing regimen (e). Depicted are the mean HbA1c values. Therapy with a Ribavirin-containing regimen leads to a nonsignificant drop of $\mathrm{Hb}$ (f). Depicted are the mean, absolute $\mathrm{Hb}$ values. 


\section{Supplementary Table II. Therapy Failure - Clinical \\ Characteristics}

\begin{tabular}{|c|c|c|c|c|c|}
\hline Genotype $\left(16^{\star}\right)$ & $A U$ & $1 \mathbf{a}$ & $1 b$ & 2 & $3 a$ \\
\hline & 16 & 3 & 5 & 1 & 7 \\
\hline Therapy experienced $\left(9^{*}\right)$ & $6 / 9$ & $0 / 0$ & $4 / 5$ & $0 / 1$ & $2 / 3$ \\
\hline Cirrhosis $\left(16^{*}\right)$ & $8 / 16$ & $3 / 3$ & $4 / 5$ & $1 / 1$ & $0 / 7$ \\
\hline $\mathrm{BMI}>25\left(6^{*}\right)$ & $6 / 6$ & $3 / 3$ & $2 / 2$ & $1 / 1$ & $0 / 0$ \\
\hline IFG $\left(15^{\star}\right)$ & $6 / 15$ & $0 / 3$ & $2 / 4$ & $0 / 1$ & $4 / 7$ \\
\hline $\begin{array}{l}\text { Baseline log10 HCV RNA } \\
\text { titre [IU/ml] MEAN (SD ) } \\
\left(10^{*}\right)\end{array}$ & - & $\begin{array}{c}6,86 \\
-\end{array}$ & $\begin{array}{c}0,9 \\
(0,8)\end{array}$ & $\begin{array}{c}0,17 \\
-\end{array}$ & $\begin{array}{c}8 \\
(7,2)\end{array}$ \\
\hline Albumin (STD) $\left(16^{*}\right)$ & - & $\begin{array}{c}36,3 \\
-\end{array}$ & $\begin{array}{c}34 \\
(3,1)\end{array}$ & $\begin{array}{c}43 \\
-\end{array}$ & $\begin{array}{c}35 \\
(5,6)\end{array}$ \\
\hline \multicolumn{6}{|l|}{ Gender $\left(16^{\star}\right)$} \\
\hline$\sigma^{\lambda}$ & 13 & 1 & 4 & 1 & 7 \\
\hline q & 3 & 2 & 1 & - & - \\
\hline
\end{tabular}

\footnotetext{
* Values marked with a star represent the number of patients with available information regarding the parameter in question.
} 